\title{
Desenvolvimento de Modelo Computacional para Previsão da Qualidade do Efluente de Lagoa Facultativa Usando a Abordagem da Dinâmica de Sistemas
}

Development of a Computational Model to Predict the Quality of Facultative Pond Effluent Using the System Dynamics Approach

Desarrollo de Modelo Computacional para Previsión de la Calidade del Efluente de Laguna Facultativa Usando el Abordaje de la Dinámica de Sistemas

Bruna Logatti

Mestranda, UFSCAR, Brasil. bru.logatti@gmail.com

Erich Kellner Professor Doutor, UFSCAR, Brasil. erich.kellner@ufscar.br 


\section{RESUMO}

Este trabalho apresenta os resultados preliminares do desenvolvimento de modelo computacional para previsão da qualidade do efluente de lagoa facultativa proposta por FRITZ (1985) com abordagem da dinâmica de sistemas, sendo utilizado o software VenSim PLE ${ }^{\circledR}$. O modelo considera o balanço de massa aplicado em um reator de mistura completa, consistindo na resolução simultânea de doze equações diferenciais no tempo para determinação das concentrações efluentes da DQO bruta, DQO solúvel, oxigênio dissolvido, Nitrogênio Orgânico e Inorgânico, Nitrogênio Total Kjeldhal, Nitrogênio Amoniacal, Fósforo Orgânico e Inorgânico, Alcalinidade, concentração hidrogeniônica $(\mathrm{pH})$. Os resultados das simulações para os parâmetros DQO bruta, DQO solúvel, Nitrogênio Total Kjeldhal e Nitrogênio Amoniacal, realizadas para o período de um ano, foram comparados com os resultados obtidos por Pedrelli (1997) para uma lagoa facultativa tratando esgoto sanitário e localizada em Balneário Camburiú (SC). Os resultados das simulações obtidos para DQO bruta e solúvel apresentarem resultados satisfatórios em relação aos obtidos em campo, porém para os demais parâmetros os resultados da simulação foram ligeiramente superiores aos observados. Tais variações podem estar associadas a não consideração da variabilidade da qualidade do afluente na lagoa facultativa, assim como a possibilidade de ocorrência de compartimentalização do volume líquido, o que afastaria o modelo da condição de mistura completa.

PALAVRAS-CHAVE: Lagoa de estabilização, modelo computacional, qualidade do efluente.

\section{ABSTRACT}

This work presents the preliminary results of the development of a computer model to predict the quality of the facultative pond effluent proposed by FRITZ (1985) with a system dynamics approach, using the software VenSim PLE ${ }^{\circledR}$. The model considers the mass balance applied in a complete mixing reactor, consisting of the simultaneous resolution of twelve differential equations in time for the determination of effluent concentrations of Crude COD, soluble COD, dissolved oxygen, Organic and Inorganic Nitrogen, Total Kjeldhal Nitrogen, Nitrogen Ammoniacal, Organic and Inorganic Phosphorus, Alkalinity, Hydrogenionic Concentration ( $p H$ ). The results of the simulations for the parameters COD, soluble COD, Total Kjeldhal Nitrogen and Ammoniacal Nitrogen, were compared with the results obtained by Pedrelli (1997) for a facultative pond treating sanitary sewage and located in Balneário Camburiú (SC). The results of the simulations obtained for crude and soluble COD presented satisfactory results in relation to those obtained in the field, but for the other parameters the simulation results were slightly higher than those observed. Such variations may be associated with a lack of consideration of the influent quality variability in the facultative pond, as well as the possibility of compartmentalization of the liquid volume, which would move the model away from the complete mixing condition.

KEYWORDS: Stabilization pond, computational model, effluent quality

\section{RESUMEN}

Este trabajo presenta los resultados preliminares del desarrollo de modelo computacional para previsión de la calidad del efluente de laguna facultativa propuesta por FRITZ (1985) con enfoque de la dinámica de sistemas, siendo utilizado el software VenSim PLE ${ }^{\circledR}$. El modelo considera el balance de masa aplicado en un reactor de mezcla completa, consistente en la resolución simultánea de doce ecuaciones diferenciales en el tiempo para determinar las concentraciones efluentes de la DQO bruta, DQO soluble, oxígeno disuelto, Nitrógeno Orgánico e Inorgánico, Nitrógeno Total Kjeldhal, Nitrógeno Amoniacal, Fósforo Orgánico e Inorgánico, Alcalinidad, concentración hidrogeniónica $(\mathrm{pH})$. Los resultados de las simulaciones para los parámetros DQO bruta, DQO soluble, Nitrógeno total Kjeldhal y Nitrógeno amoniacal, realizados para el período de un año, fueron comparados con los resultados obtenidos por Pedrelli (1997) para una laguna facultativa tratando alcantarillado sanitario y ubicada en Balneario Camburiú (SC). Los resultados de las simulaciones obtenidas para DQO bruta y soluble presentan resultados satisfactorios en relación a los obtenidos en campo, pero para los demás parámetros los resultados de la simulación fueron ligeramente superiores a los observados. Tales variaciones pueden estar asociadas a la no consideración de la variabilidad de la calidad del afluente en la laguna facultativa, así como la posibilidad de ocurrencia de compartimentalización del volumen líquido, lo que alejará el modelo de la condición de mezcla completa.

PALABRAS CLAVE: Laguna de estabilización, modelo computacional, calidad del efluente. 


\section{INTRODUÇÃO}

Conforme o Sistema Nacional de Informações sobre Saneamento (SNIS), 50,3\% da população brasileira têm acesso à coleta de esgoto, ou seja, mais de 100 milhões não tem acesso a esse serviço. Além disso, apenas 42,67\% do esgoto do país é tratado. (BRASIL, 2017). Dados de 2016 do Instituto Brasileiro de Geografia e Estatística (IBGE) indicam que o Brasil possui 5570 municípios, dos quais $68,38 \%$ apresentam menos de 20.000 habitantes e $94,33 \%$ apresentam menos que 100.000 habitantes. (IBGE, 2016). Portanto, o Brasil é constituído de pequenos municípios que não apresentam tratamento de esgoto.

Tais dados são importantes para a determinar o tipo de tratamento de esgoto a ser adotado dentro desses municípios. Levando-se em consideração o tamanho das cidades brasileiras, o clima apresentado pelo país e a disponibilidade de espaço e recursos para tal atividade é inevitável que as lagoas de estabilização estejam entre as opções mais viáveis para o tratamento de esgoto.

As lagoas de estabilização são um dos métodos mais simples para tratamento de efluentes, elas possuem uma fácil construção, além disso, sua manutenção e operação não possuem grandes desafios, também não necessitam de mão-de-obra especializada e equipamentos mecânicos (quando bem projetadas). São altamente recomendadas para locais que apresentam clima tropical (altas temperaturas e incidência de raios solares), já que o tratamento é muito dependente de reações bioquímicas. (VON SPERLING, 2007)

As lagoas de estabilização podem ser anaeróbias, facultativas ou de maturação. As lagoas facultativas são responsáveis pelo tratamento secundário dos esgotos, possuem como principal característica a dualidade ambiental: aeróbia na superfície e anaeróbia no fundo. (KELLNER e PIRES, 1998).

Na parte mais funda das lagoas facultativas, a matéria orgânica é estabilizada por processos anaeróbios. Nessa região, a matéria orgânica é convertida em metano $\left(\mathrm{CH}_{4}\right)$ e dióxido de carbono $\left(\mathrm{CO}_{2}\right)$, os quais são disponibilizados para a fase líquida. Na porção superior da lagoa facultativa, prevalece a condição aeróbia. Essa região é responsável pela estabilização da matéria orgânica por processo aeróbio, ocorrendo a oxidação dos compostos reduzidos produzidos na região anaeróbia, minimizando a liberação de odores (GRADY JR. et al., 2011).

Em 1985 o cientista J. J. Fritz desenvolveu um modelo matemático capaz de simular as reações bioquímicas existentes dentro das lagoas facultativas. O modelo se baseia nos princípios da conservação de massas em um reator de mistura completa e consiste na resolução de 12 equações diferenciais que representam as variações temporais da biomassa ou de substâncias químicas presentes na lagoa de estabilização. (FRITZ, 1985).

O presente trabalho resultou da implementação das equações diferenciais aplicando a abordagem da dinâmica de sistemas, sendo utilizado o software VenSim PLE ${ }^{\circledR}$. Os resultados mostraram-se satisfatórios, indicando, porém, a necessidade de consideração da variabilidade do afluente, assim como da estratificação da coluna líquida. 


\section{OBJETIVOS}

O objetivo deste trabalho foi o de desenvolver um modelo computacional no software VenSim $^{\circledast}$ capaz de simular as reações bioquímicas e as iterações que ocorrem nas lagoas facultativas a partir do modelo matemático proposto por Fritz (1985).

\section{METODOLOGIA/MÉTOdO DE ANÁLISE}

\subsection{Modelo de Fritz (1985)}

A técnica básica do modelo matemático de Fritz (1985) é a de desenvolver simultaneamente diversas equações de conservação de massa para as várias espécies bioquímicas (DBO, algas, $\mathrm{OD}$, entre outras) com o fim de simular as reações biológicas existentes dentro das lagoas facultativas. O modelo considera a lagoa como uma mistura completa. (FRITZ, 1985).

As equações de conservação de massa desenvolvem-se para espécies químicas e biomassa reagindo a fatores ambientais que afetam os mecanismos dos processos. Para essas equações dispõem-se das concentrações dos afluentes e efluentes, sendo eles: demanda química de oxigênio (DQO), oxigênio dissolvido, bactérias, algas, carbono inorgânico, fósforo orgânico e inorgânico, nitrogênio orgânico, amônia, nitrato, alcalinidade e formação de lodo (Quadro 1). (FRITZ, 1985).

O modelo simula as características das lagoas como uma função do tempo, portanto está sujeito a fatores físicos e ambientais específicos do local para o período de tempo selecionado (dia ou mês). As taxas de reações (Quadro 2) que são responsáveis pelo equilíbrio entre os balanços das concentrações, também são dependentes desses fatores ambientais. (FRITZ, 1985). 


\section{Revista Nacional de}

Quadro 1: Equações de Conservação de Massa para Modelo de Fritz (1985).

\begin{tabular}{|l|c|}
\hline \multicolumn{1}{|c|}{ Tipo } & Equação \\
\hline $\begin{array}{l}\text { Demanda Química de Oxigênio } \\
\mathrm{DQO}(\mathrm{mg} / \mathrm{l})\end{array}$ & \multicolumn{1}{c|}{$\frac{d S}{d t}=\frac{S i s}{\theta}-\frac{S}{\theta}+r s$} \\
\hline $\begin{array}{l}\text { Concentração da Biomassa Bacteriana } \\
\mathrm{Xb}(\mathrm{mg} / \mathrm{l})\end{array}$ & $\frac{d X_{b}}{d t}=\frac{X_{b i}}{\theta}-\frac{X_{b}}{\theta}+r x_{b 1}+r x_{b 2}$ \\
\hline $\begin{array}{l}\text { Concentração de Algas } \\
\text { Xa (mg/l) }\end{array}$ & $\frac{d X_{a}}{d t}=\frac{X_{a i}}{\theta}-\frac{X_{a}}{\theta}+r x_{a 1}+r x_{a 2}$ \\
\hline $\begin{array}{l}\text { Concentração de Oxigênio } \\
\mathrm{O}_{2}(\mathrm{mg} / \mathrm{l})\end{array}$ & $\frac{d O_{2}}{d t}=\frac{O_{2 i}}{\theta}-\frac{O_{2}}{\theta}+r 1_{d o}+r 2_{d o}+r 3_{d o}+r 4_{d o}$ \\
\hline $\begin{array}{l}\text { Concentração Nitrogênio Orgânico } \\
\mathrm{No}(\mathrm{mg} / \mathrm{l})\end{array}$ & $\frac{d N_{o}}{d t}=\frac{N_{o i}}{\theta}-\frac{N_{o}}{\theta}+r 1_{n o}+r 2_{n o}$ \\
\hline $\begin{array}{l}\text { Concentração de Amônia } \\
\mathrm{NH} \text { (mg/l) }\end{array}$ & $\frac{N N H_{4}}{d t}=\frac{N H_{4 i}}{\theta}-\frac{N H_{4}}{\theta}+r 1_{a m}+p 1 r 2_{a m}+r 3_{a m}+r 4_{a m}$ \\
\hline $\begin{array}{l}\text { Concentração de Nitrato } \\
\mathrm{NO} \text { (mg/l) }\end{array}$ & $\frac{d N O_{3}}{d t}=\frac{N O_{3 i}}{\theta}-\frac{N O_{3}}{\theta}+r_{1 n a}+p 2 r_{2 n a}$ \\
\hline $\begin{array}{l}\text { Concentração Fósforo Orgânico } \\
\text { Po (mg/l) }\end{array}$ & $\frac{d P_{o}}{d t}=\frac{P_{o i}}{\theta}-\frac{P_{o}}{\theta}+r_{1 o p}+r_{2 o p}$ \\
\hline $\begin{array}{l}\text { Concentração Fósforo Inorgânico } \\
\text { Pi (mg/l) }\end{array}$ & $\frac{d P_{i}}{d t}=\frac{P_{i i}}{\theta}-\frac{P_{i}}{\theta}+r_{1 i p}+r_{2 i p}+r_{3 i p}$ \\
\hline $\begin{array}{l}\text { Concentração de Carbono Inorgânico } \\
\text { Total Ct (mg/l) }\end{array}$ & $\frac{d C_{t}}{d t}=\frac{C_{t i}}{\theta}-\frac{C_{t}}{\theta}+r_{1 c t}+r_{2 c t}+r_{3 c t}+r_{4 c t}$ \\
\hline $\begin{array}{l}\text { Alcalinidade } \\
\text { ALK (mg/l) }\end{array}$ & \begin{tabular}{l}
$\frac{d A L K}{d t}=\frac{A L K i}{\theta}-\frac{A L K}{\theta}+r 1 a$ \\
\hline $\begin{array}{l}\text { Lodo Gerado } \\
\text { Dm (mg/l) }\end{array}$
\end{tabular} \\
\hline
\end{tabular}

Fonte: adaptado de FRITZ (1985).

Quadro 2: Taxas de Reação para Balanço da Massa.

\begin{tabular}{|c|c|c|}
\hline Reação & Taxa de Reação Volumétrica & \\
\hline \multicolumn{3}{|l|}{ Crescimento Bacteriano } \\
\hline Utilização da DQO degradável & $r s=-k * X b \cdot\left[\frac{S}{K S+S}\right] *\left[\frac{O_{2}}{K O_{2}+O_{2}}\right] * *\left[\frac{N_{i}}{K b_{N}+N_{i}}\right] *\left[\frac{P i}{K b_{P}+P i}\right]$ & (13) \\
\hline Crescimento das bactérias & $r x b 1=-Y * r s-k b * X b$ & (14) \\
\hline Sedimentação & $r \times b 2=-s b * X b$ & (15) \\
\hline \multicolumn{3}{|l|}{ Crescimento de Algas } \\
\hline Crescimento de algas & $\begin{aligned} r x a 1=X a *\{\mu a & * f(L) * f(T) *\left[\frac{C O_{2}}{K C O_{2}+C O_{2}}\right] * *\left[\frac{N_{i}}{K a_{N}+N_{i}}\right] \\
& \left.*\left[\frac{P i}{K a_{P}+P i}\right]-k a\right\}\end{aligned}$ & (16) \\
\hline Sedimentação & $r x a 2=-s a * X a$ & (17) \\
\hline \multicolumn{3}{|l|}{ Geração e Utilização de Oxigênio } \\
\hline Transferência interfacial & $r 1 o d=\frac{A}{V} * K 1 O_{2} *\left(O_{\text {sat }}-O_{2}\right)$ & (18) \\
\hline Fotossíntese & $r 2 o d=1,244 * r \times a 1$ & (19) \\
\hline Decaimento bacteriano & $r 3 o d=-[(1-1,42 * Y) *(-r s)+1,42 * k b * X b]$ & $(20)$ \\
\hline
\end{tabular}




\section{Revista Nacional de}

\begin{tabular}{|c|c|c|}
\hline Nitrificação & $r 4 o d=-4,57 * r 3 a m$ & (21) \\
\hline \multicolumn{3}{|l|}{ Alcalinidade } \\
\hline Nitrificação & $r 1 a=-7,14 * r 3 a m$ & (22) \\
\hline \multicolumn{3}{|l|}{ Nitrogênio Orgânico } \\
\hline $\begin{array}{l}\text { Decaimento de bactérias e } \\
\text { algas }\end{array}$ & $r 1$ no $=0,124 * k b * X b+0,063 * k a * X a$ & (23) \\
\hline $\begin{array}{l}\text { Transformação do N-Orgânico } \\
\text { em NH3 }\end{array}$ & $r 2 n o=-\alpha_{N} * N_{O}$ & (24) \\
\hline \multicolumn{3}{|l|}{ Amônia } \\
\hline $\begin{array}{l}\text { Transformação do N-Orgânico } \\
\text { em NH3 }\end{array}$ & $r 1 a m=-r 2 n o$ & (25) \\
\hline $\begin{array}{l}\text { Crescimento de algas e } \\
\text { bactérias }\end{array}$ & $r 2 a m=0,063 *(r x a 1+k a * X a)-0,124 * *(-Y * r s)$ & (26) \\
\hline Nitrificação & $r 3 a m=-\frac{\mu_{N}}{Y_{N}} *\left[\frac{N H_{4}}{K_{N}+N H_{4}}\right] *\left[\frac{O_{2}}{K N O_{2}+O_{2}}\right] *[C p H] *\left[C_{T}\right]$ & (27) \\
\hline Regeneração bêntica & $r 4 a m=\frac{R_{N}}{h}$ & (28) \\
\hline \multicolumn{3}{|l|}{ Nitrato } \\
\hline Nitrificação & $r 1 n a=r 3 a m$ & (29) \\
\hline $\begin{array}{l}\text { Crescimento de algas e } \\
\text { bactérias }\end{array}$ & $r 2 n a=r 2 a m$ & (30) \\
\hline \multicolumn{3}{|l|}{ Fósforo Orgânico } \\
\hline $\begin{array}{l}\text { Decaimento de algas e } \\
\text { bactérias }\end{array}$ & $r 1 o p=0,009 * k a * X a+0,024 * k b * X b$ & (31) \\
\hline $\begin{array}{l}\text { Transformação do P-orgânico } \\
\text { em P-inorgânico }\end{array}$ & $r 2 o p=-\alpha_{P} * P_{O}$ & (32) \\
\hline \multicolumn{3}{|l|}{ Fósforo Inorgânico } \\
\hline $\begin{array}{l}\text { Crescimento de algas e } \\
\text { bactérias }\end{array}$ & $r 1 i p=0,009 *(r x a 1+k a * X a)-0,024 * *(-Y * r s)$ & (33) \\
\hline $\begin{array}{l}\text { Transformação do P-orgânico } \\
\text { em P-inorgânico }\end{array}$ & $r 2 i p=r 2 p o$ & (34) \\
\hline Regeneração bêntica & $r 3 i p=\frac{R_{P}}{h}$ & (35) \\
\hline \multicolumn{3}{|c|}{ Geração e Utilização do Carbono Inorgânico Total } \\
\hline Transferência interfacial & $r 1 c t=\frac{12}{44} * \frac{A}{V} * K_{1 C O} *\left(\mathrm{CO}_{2 s a t}-\mathrm{CO}_{2}\right)$ & (36) \\
\hline Decaimento bacteriano & $r 2 c t=\frac{12}{32} * r 3 o d$ & $(37)$ \\
\hline Fotossíntese & $r 3 c t=-1,314 * r x a 1$ & (38) \\
\hline Decaimento bentônico & $r 4 c t=\frac{R_{C} * C_{M}}{h}$ & (39) \\
\hline \multicolumn{3}{|l|}{ Lodo Anaeróbio } \\
\hline Sedimentação & $r 1 d=\frac{1}{h} *(s a * X a+s b * X b)$ & (40) \\
\hline Ressuspensão & $r 2 d=U r * D$ & (41) \\
\hline
\end{tabular}

Fonte: adaptado de FRITZ (1985). 
Revista Nacional de

\subsection{Modelo Computacional}

O modelo matemático de Fritz pode ser descrito como um conjunto de equações diferenciais que para serem resolvidas analiticamente necessitarão de demasiado tempo e conhecimento. Portanto, para se obter uma solução é necessário um meio de discretizar as equações e tentar aproximá-las de um sistema de equações algébricas, ou seja, deve ser usado um modelo computacional. (FERZIGER, PERIC, 2002).

O papel do modelo é de aplicar aproximações a pequenos domínios no espaço e/ou tempo, possibilitando uma solução numérica com resultados em locais distintos no espaço e no tempo. Apesar da precisão dos dados experimentais ser dependente da qualidade das ferramentas utilizadas, quando se tem soluções numéricas, elas são dependentes das discretizações usadas. (FERZIGER, PERIC, 2002).

O modelo matemático de Fritz necessitava de uma plataforma que permitisse:

$>$ Iterações dinâmicas;

$>$ Retroalimentação;

$>$ Resolução de equações diferenciais interdependentes;

$>$ Geração de gráficos;

$>$ Variáveis de entrada dependentes de variáveis de saída.

Tendo em vista todos esses itens o Software VenSim $\mathrm{PLE}^{\circledR}$ tornou-se uma ótima ferramenta para desenvolvimento do modelo. Já que cumpre a maior parte dos tópicos com o adicional de possuir uma interface de fácil compreensão e desenvolvimento.

\subsubsection{VenSim PLE $^{\circledR}$}

Software elaborado pela Ventana Systems, Inc., tem como principal objetivo melhorar o desempenho de sistemas reais. Ele é utilizado para desenvolver, analisar e empacotar modelos de feedback dinâmico. O Vensim PLE ${ }^{\circledast}$ (Personal Learning Edition) tem como função introduzir o usuário ao sistema de modelos dinâmicos, ele é gratuito para o uso educacional e acadêmico. A principal função do software é o Causal Tracing TM que permite descobrir quais coisas estão fazendo com que outras mudem.

Ele se destaca pela simplicidade na construção de modelos, utilizando diagramas causais ou diagramas de estoques e taxas (GONÇALVES, 2009). O modelo computacional (Figura 2) foi construído com base no diagrama de blocos do modelo matemático (Figura 1). Observa-se que as lagoas facultativas são sistemas de simples operação e manutenção, entretanto de extrema complexidade quando diz respeito às suas reações.

\subsection{Calibração do Modelo}




\section{Revista Nacional de}

A fim de analisar a validade do modelo desenvolvido, necessita-se que o mesmo seja testado diante de dados reais obtidos em uma lagoa facultativa. Para isso, utilizou-se dados coletados na dissertação de mestrado de Pedrelli (1997). Porém nem todos os dados necessários para o funcionamento apropriado do modelo apresentam-se no trabalho, estes tiveram que ser adotados com base em outras lagoas.

\subsubsection{Concentração dos Afluentes}

Para o modelo computacional desenvolvido os dados usados para calibragem são os obtidos na saída da lagoa anaeróbia (L. A.), portanto efluente da lagoa anaeróbia e afluente da lagoa facultativa. Esses dados são apresentados na Tabela 3, com seus respectivos nomes, valores e fonte. O trabalho não apresentava a concentração de algas e bactérias (Xai e Xbi), a concentração do carbono inorgânico total (Cti) e a concentração de fósforo orgânico e inorgânico (Pii e Poi), portanto elas foram adotadas de outro referencial bibliográfico que apresentado na Tabela 3.

Figura 1: Diagrama de bloco representando as iterações bioquímicas consideradas no modelo de Fritz (1985).

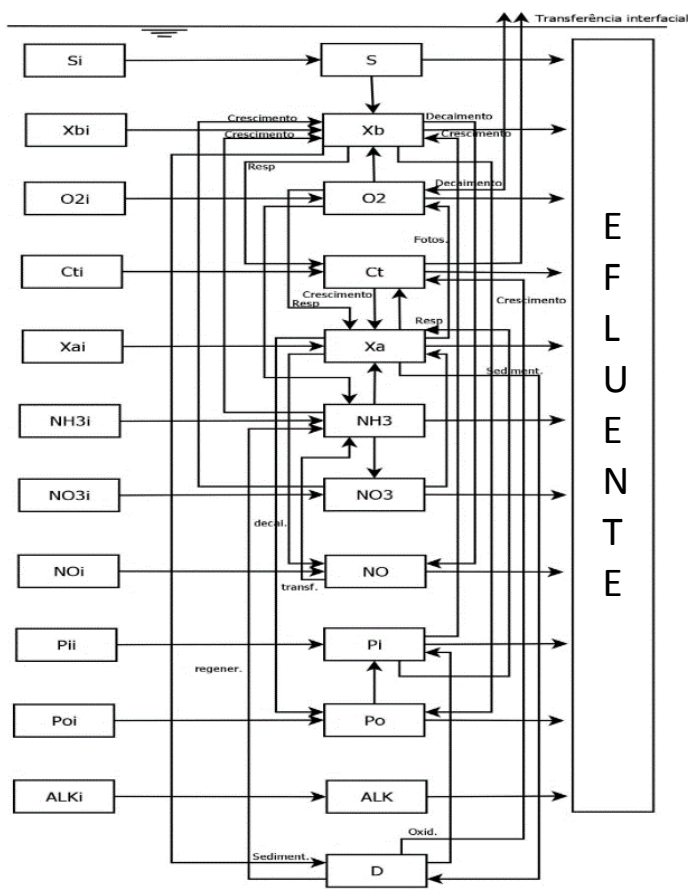

Fonte: FRITZ, 1985. 
Revista Nacional de Gerenciamento de Cidades National Journal of Cities Management

Figura 2: Modelo de Fritz (1985) desenvolvido dentro da Plataforma VenSim PLE ${ }^{\circledR}$

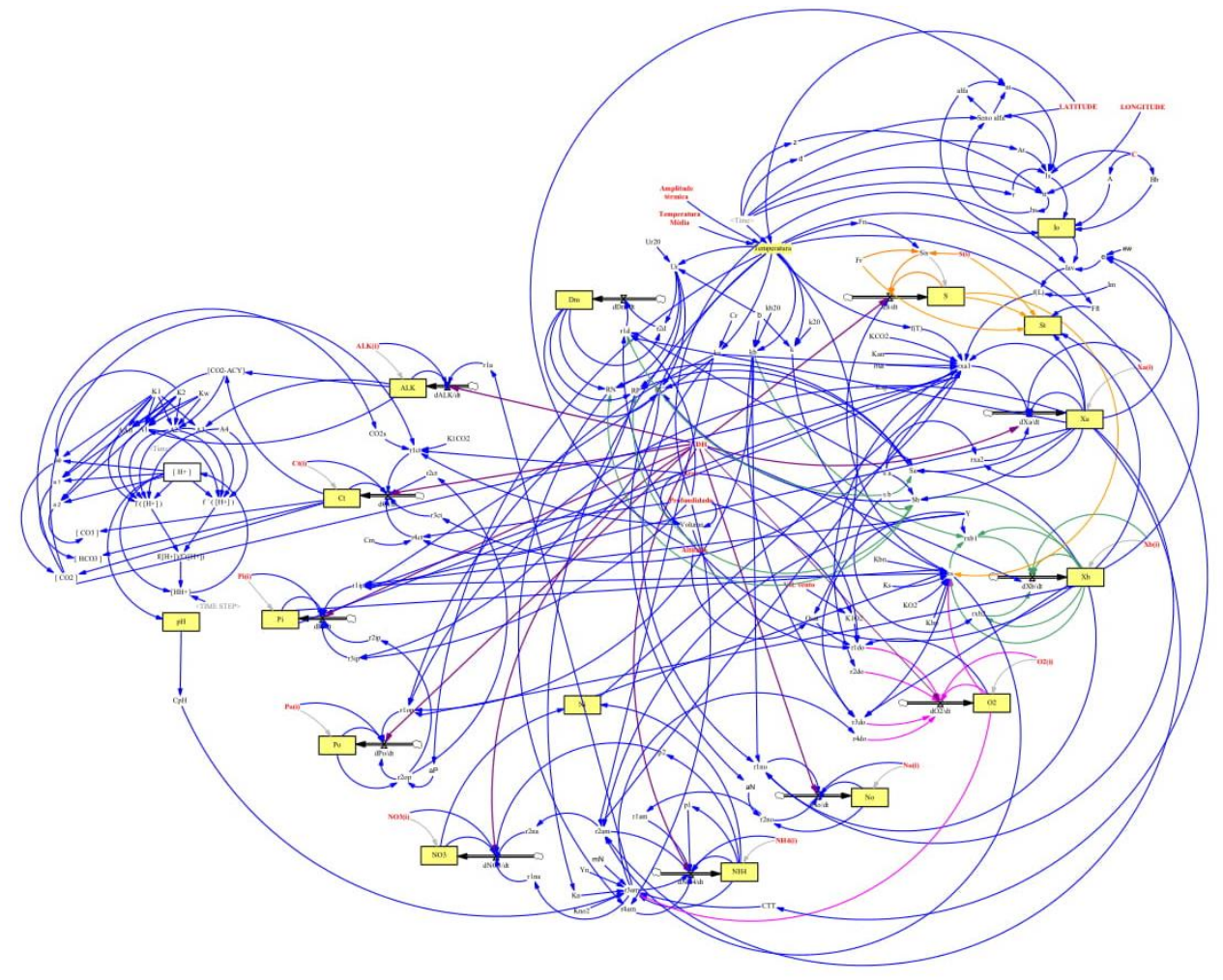

Fonte: AUTORES, 2018.

Tabela 3: Resultados Operacionais da Lagoa Anaeróbia.

\begin{tabular}{ccc}
\hline Afluente & Valor $(\mathrm{mg} / \mathrm{l})$ & Fonte \\
\hline $\mathrm{Si}$ & 210 & PEDRELLI, 1997 \\
$\mathrm{Xbi}$ & 1 & FRITZ, 1985 \\
$\mathrm{Xai}$ & 1 & FRITZ, 1985 \\
$\mathrm{O}_{2} \mathrm{i}$ & 0 & PEDRELLI, 1997 \\
$\mathrm{Noi}$ & 8,48 & PEDRELLI, 1997 \\
$\mathrm{NH}_{4} \mathrm{i}$ & 35,35 & PEDRELLI, 1997 \\
$\mathrm{NO}_{3} \mathrm{i}$ & 0,45 & PEDRELLI, 1997 \\
$\mathrm{Poi}$ & 3,05 & TRUPPEL, 2002 \\
$\mathrm{Pii}$ & 6,09 & TRUPPEL, 2002 \\
$\mathrm{Cti}$ & 60 & FRITZ, 1985 \\
$\mathrm{ALKi}$ & 238 & PEDRELLI, 1997 \\
\hline
\end{tabular}

\subsubsection{Coeficiente Bioquímicos}

A Tabela 4 apresenta os coeficientes bioquímicos utilizados no modelo, os coeficientes foram obtidos por meio de pesquisa bibliográfica, onde a maior parte dos valores foram retirados diretamente do trabalho de Fritz (1985). 


\section{Revista Nacional de}

A utilização desses coeficientes se relaciona com a calibração do modelo. Portanto, para tal foi adotado o valor típico apresentado na Tabela 4, a partir dos resultados houveram as mudanças conforme o intervalo de valores possíveis (valor mínimo e máximo) para o mais próximo do valor real.

Tabela 4: Intervalo de Valores de Constantes Bioquímicas presentes no modelo computacional.

\begin{tabular}{|c|c|c|c|c|c|c|}
\hline \multirow{2}{*}{ Coeficiente } & \multirow{2}{*}{ Nome do Coeficiente } & \multicolumn{2}{|c|}{ Intervalo de Valores } & \multirow{2}{*}{$\begin{array}{l}\text { Valor } \\
\text { Típico }\end{array}$} & \multirow{2}{*}{ Unidade } & \multirow{2}{*}{ Fonte } \\
\hline & & Mínimo & Máximo & & & \\
\hline Y & $\begin{array}{l}\text { Coeficiente de produção } \\
\text { carbonácea }\end{array}$ & 0,4 & 0,8 & 0,6 & $\begin{array}{l}\text { mg VSS/ } \\
\text { mg DBO }\end{array}$ & pg. 585 (METCALF \& \\
\hline Y & $\begin{array}{l}\text { Coeficiente de produção } \\
\text { carbonácea }\end{array}$ & 0,3 & 0,6 & 0,4 & $\begin{array}{c}\text { mg VS/ } \\
\text { mg bsDQO }\end{array}$ & EDDY, 2003) \\
\hline $\mathrm{K} 20$ & $\begin{array}{c}\text { Taxa de máxima utilização } \\
\text { do substrato para } 20^{\circ} \mathrm{C}\end{array}$ & 2 & 10 & 5 & $\mathrm{dia}^{-1}$ & $\begin{array}{l}\text { pg. } 585 \text { (METCALF \& } \\
\text { EDDY, 2003) }\end{array}$ \\
\hline Ks & $\begin{array}{c}\text { Constante de meia } \\
\text { saturação do Substrato }\end{array}$ & 25 & 100 & 60 & $\mathrm{mg} / \mathrm{l} \mathrm{DBO}$ & pg. 585 (METCALF \& \\
\hline Ks & $\begin{array}{c}\text { Constante de meia } \\
\text { saturação do Substrato }\end{array}$ & 10 & 60 & 40 & $\begin{array}{c}\text { mg/ } \\
\text { I bsDQO }\end{array}$ & EDDY, 2003) \\
\hline $\mathrm{Kb} 20$ & $\begin{array}{c}\text { Coeficiente de decaimento } \\
\text { bacteriano }\end{array}$ & 0,25 & 0,4 & 0,35 & $\mathrm{dia}^{-1}$ & $\begin{array}{c}\text { pg. } 29 \text { (VON SPERLING, } \\
\text { 2007) }\end{array}$ \\
\hline $\mathrm{KO}_{2}$ & $\begin{array}{l}\text { Constante de meia } \\
\text { saturação do } \mathrm{O}_{2}\end{array}$ & 0,08 & 1,1 & 1,1 & $\mathrm{mg} / \mathrm{l}$ & $\begin{array}{l}\text { pg. } 434 \text { (MARA, } \\
\text { HORAN, 2003) }\end{array}$ \\
\hline Kbn & $\begin{array}{l}\text { Coeficiente de meia } \\
\text { saturação N-bacterial }\end{array}$ & - & - & 0,01 & $\mathrm{mg} / \mathrm{l}$ & pg. 212 (FRITZ, 1985) \\
\hline Kbp & $\begin{array}{l}\text { Constante de meia } \\
\text { saturação para P-bacterial }\end{array}$ & - & - & 0,01 & $\mathrm{mg} / \mathrm{l}$ & pg. 212 (FRITZ, 1985) \\
\hline$\beta$ & $\begin{array}{l}\text { Constante de } \\
\text { Arrhenius }\end{array}$ & 1,05 & 1,085 & 1,07 & - & $\begin{array}{c}\text { pg. } 30 \text { (VON SPERLING, } \\
\text { 2007) }\end{array}$ \\
\hline sb & $\begin{array}{c}\text { Taxa de sedimentação } \\
\text { bacteriana }\end{array}$ & - & - & 0,05 & $\mathrm{dia}^{-1}$ & $\begin{array}{l}\text { pg.212 (CANALE, } 1976 \\
\text { apud FRITZ, 1985) }\end{array}$ \\
\hline$\mu а$ & $\begin{array}{l}\text { Taxa de máximo } \\
\text { crescimento algal }\end{array}$ & - & - & 2,0 & $\mathrm{dia}^{-1}$ & $\begin{array}{l}\text { pg.212 (CANALE, } 1976 \\
\text { apud FRITZ, 1985) }\end{array}$ \\
\hline $\mathrm{KCO}_{2}$ & $\begin{array}{l}\text { Constante de meia } \\
\text { saturação do } \mathrm{CO}_{2}\end{array}$ & - & - & 1,0 & $\mathrm{mg} / \mathrm{l}$ & $\begin{array}{l}\text { pg.212 (GOLDMAN, } \\
1974 \text { apud FRITZ, 1985) }\end{array}$ \\
\hline Kan & $\begin{array}{l}\text { Constante de meia } \\
\text { saturação para o } \mathrm{N}_{2} \text { algal }\end{array}$ & - & - & 0,10 & $\mathrm{mg} / \mathrm{l}$ & $\begin{array}{l}\text { pg.212 (CANALE, } 1976 \\
\text { apud FRITZ, 1985) }\end{array}$ \\
\hline Kap & $\begin{array}{l}\text { Constante de meia } \\
\text { saturação para P-algal }\end{array}$ & - & - & 0,02 & $\mathrm{mg} / \mathrm{l}$ & $\begin{array}{l}\text { pg.212 (CANALE, } 1976 \\
\text { apud FRITZ, 1985) }\end{array}$ \\
\hline
\end{tabular}




\section{Revista Nacional de}

\begin{tabular}{|c|c|c|c|c|c|c|}
\hline Ка & $\begin{array}{l}\text { Coeficiente de respiração } \\
\text { algal }\end{array}$ & - & - & 0,02 & $\mathrm{dia}^{-1}$ & $\begin{array}{l}\text { pg.212 (DITORO, } 1970 \\
\text { apud FRITZ, 1985) }\end{array}$ \\
\hline$\alpha \mathrm{N}$ & $\begin{array}{l}\text { Taxa de transformação do } \\
\text { Nitrogênio orgânico }\end{array}$ & - & - & 0,08 & $\mathrm{dia}^{-1}$ & $\begin{array}{c}\text { pg.212 (DITORO, } 1970 \\
\text { apud FRITZ, 1985) }\end{array}$ \\
\hline$\mu \mathrm{N}$ & $\begin{array}{c}\text { Taxa de crescimento das } \\
\text { Nitrosomonas }\end{array}$ & 0,002 & 0,008 & 0,008 & $\mathrm{dia}^{-1}$ & pg. 215 (FRITZ, 1985) \\
\hline $\mathrm{Yn}$ & $\begin{array}{l}\text { Coeficiente de produção } \\
\text { de Nitrosomonas }\end{array}$ & - & - & 0,15 & $\mathrm{mg} / \mathrm{mg}$ & pg. 213 (FRITZ, 1985) \\
\hline$\alpha p$ & $\begin{array}{l}\text { Taxa de transferência para } \\
\text { Fósforo orgânico }\end{array}$ & 0,002 & 0,02 & 0,02 & $\mathrm{dia}^{-1}$ & $\begin{array}{l}\text { pg.217 (DITORO, } 1970 \\
\text { apud FRITZ, 1985) }\end{array}$ \\
\hline $\mathrm{Cm}$ & $\begin{array}{l}\text { Taxa dióxido de } \\
\text { carbono/carbono }\end{array}$ & - & - & 0,5 & - & pg. 213 (FRITZ, 1985) \\
\hline sa & $\begin{array}{c}\text { Taxa de sedimentação } \\
\text { algal }\end{array}$ & - & - & 0,05 & $\mathrm{dia}^{-1}$ & $\begin{array}{c}\text { pg.212 (CANALE, } 1976 \\
\text { apud FRITZ, 1985) }\end{array}$ \\
\hline Ur20 & $\begin{array}{c}\text { Taxa de regeneração } \\
\text { bêntica para } 20^{\circ} \mathrm{C}\end{array}$ & - & - & 0,09 & $\mathrm{dia}^{-1}$ & $\begin{array}{l}\text { pg.212 (FOREE, } 1970 \\
\text { apud FRITZ, 1985) }\end{array}$ \\
\hline
\end{tabular}

\subsubsection{Simulações}

Para a calibração foram feitas três simulações, em todas foram adotadas as concentrações do afluente mostradas na Tabela 3. Para cada simulação era inserido um conjunto de valores diferentes das constantes bioquímicas (Tabela 4). A primeira simulação (Coeftipico) consistia dos valores típicos, a segunda (CoefMinimo) dos valores mínimos e a terceira (CoefMaximo) dos valores máximos.

\section{RESULTADOS}

Para os resultados fez-se uma análise comparativa entre os gráficos obtidos como resposta final do modelo e os dados obtidos através de amostras coletadas na lagoa facultativa estudada por Pedrelli (1997). Será feito esse estudo comparativo relativo com as amostras de DQO, DQO solúvel, NTK e $\mathrm{NH}_{4}$.

A terceira simulação com os valores máximos apresentou erros na renderização e os gráficos mostrados pelo VenSim $^{\circledR}$ estavam bem diferentes das curvas obtidas na simulação 1 e 2. Portanto, a curva da terceira simulação foi descartada dos gráficos comparativos finais.

Tendo em vista que o modelo considera uma concentração de afluente constante e na realidade a concentração varia conforme o tempo, determinou-se que a partir das curvas obtidas nas simulações 1 e 2 deveriam ser criadas outras duas curvas, uma aumentando em 


\section{Revista Nacional de}

$10 \%$ (1.1CoefTipico) e outra reduzindo em 10\% (0.9CoefTipico) a curva CoefTipico, a fim de que a análise seja mais condizente com uma lagoa real.

\subsection{Demanda Química de Oxigênio - DQO (S)}

A DQO é apresentada no modelo como S e é calculada com base na Equação (1) do Quadro 1. As curvas geradas no modelo apresentam-se no Gráfico 1.

Gráfico 1: Curvas da DQO (S) obtida no Modelo Computacional de Fritz (1985).

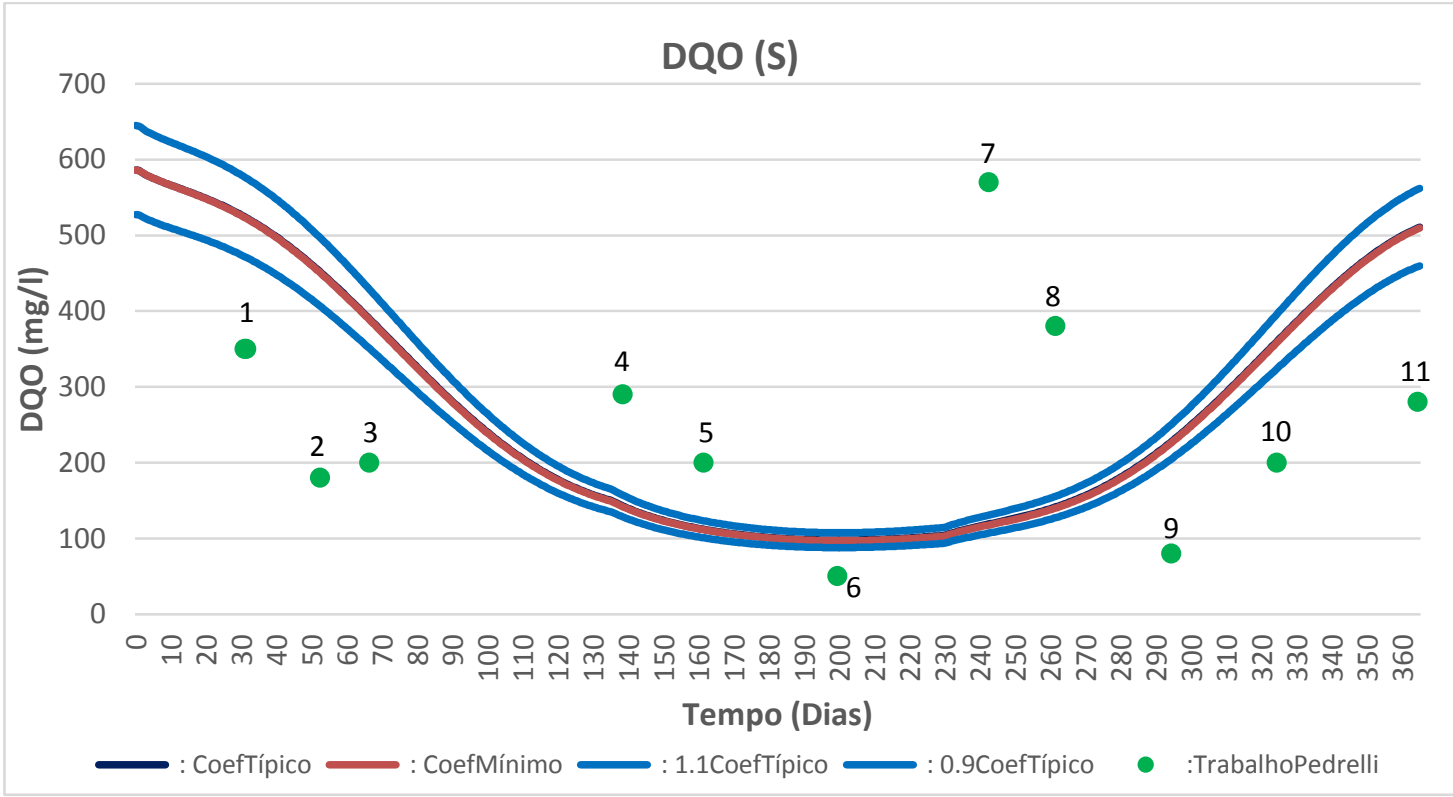

Fonte: AUTORES, 2018

No Gráfico 1 pode-se observar que as curvas do Coeficiente Típico e do Coeficiente Mínimo não apresentam diferença visual, além disso dos onze pontos obtidos do trabalho de Pedrelli (1997), nenhum se encontra dentro do intervalo estipulado entre a curva 0.9CoefTípico e 1.1CoefTípico.

Dos onze pontos obtidos, quatro encontram-se acima do conjunto de curvas e sete abaixo. Indicando que em $63,3 \%$ das amostras colhidas, a concentração real de DQO do efluente é menor do que a obtida para o mesmo dia no modelo.

\subsection{Demanda Química de Oxigênio Solúvel - DQO Solúvel (St)}

A DQO Solúvel é apresentada no modelo como St. No Gráfico 2 são apresentadas as curvas geradas. 


\section{Revista Nacional de}

Gráfico 2: Curvas da DQO Solúvel (St) obtida no Modelo Computacional de Fritz (1985).

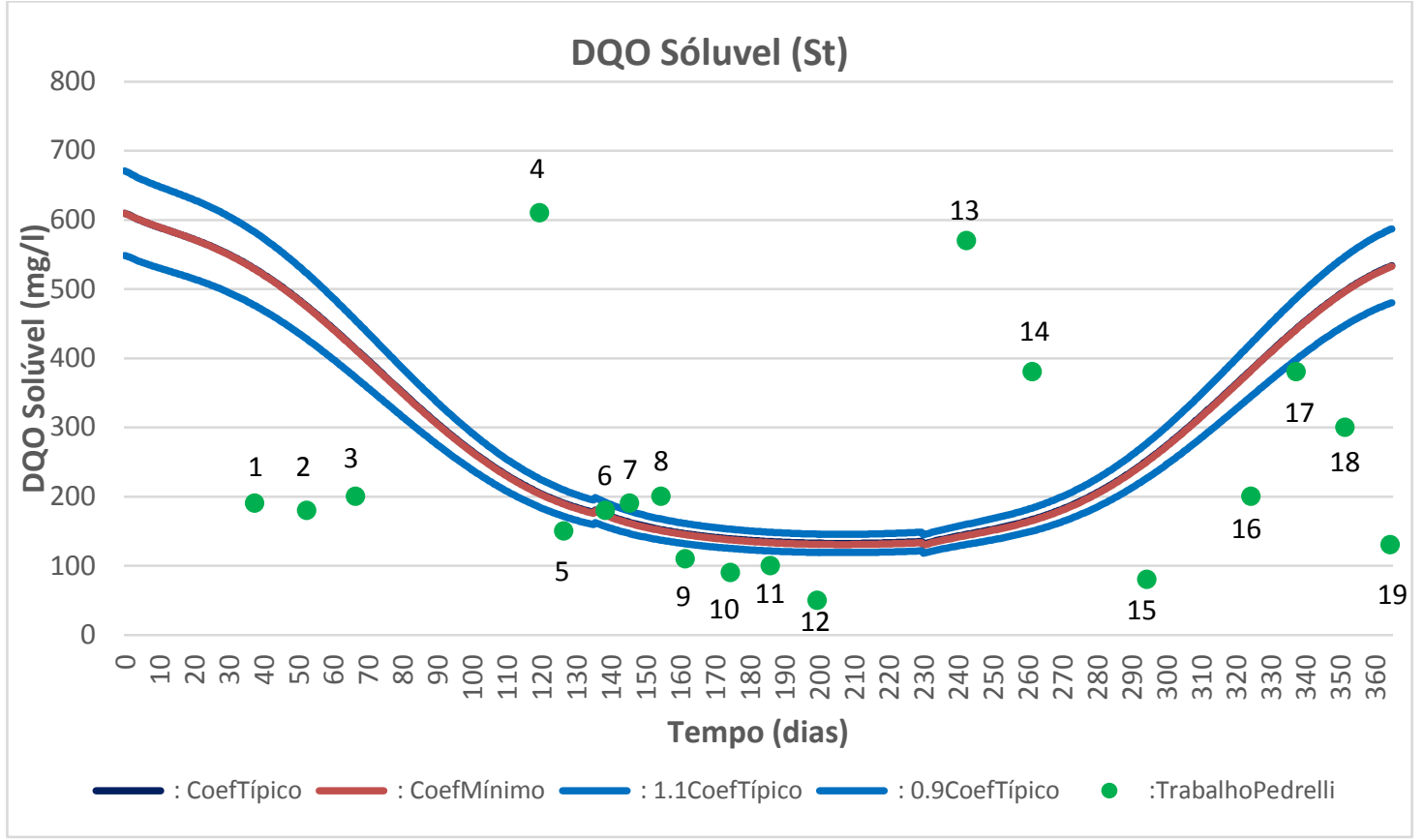

Fonte: AUTORES, 2018

Observando o Gráfico 2, chega-se a mesma conclusão obtida no Gráfico 1: as curvas Coeficiente Típico e Coeficiente Mínimo não apresentam diferença visual. Dos 19 pontos obtidos da lagoa real, 1 (um) encontra-se dentro do intervalo das curvas (Ponto 6), 3 (três) pelo menos o tocam (Pontos 7,11 e 17) e 2 (dois) estão muito próximos (Pontos 5 e 9). Portanto, $21,1 \%$ dos pontos analisados possuem algum tipo de contato com o conjunto e $10,5 \%$ apresentam-se bem próximos dos resultados obtidos pelo modelo.

Dos 13 pontos restantes, 9 encontram-se abaixo do conjunto e 4 acima, ou seja, em 47,4\% das amostras colhidas a concentração real de DQO solúvel do efluente é menor do que a obtida para o mesmo dia no modelo.

\subsection{Concentração de Nitrogênio Total Kieldhal - NTK}

O NTK foi calculado através da soma do $\mathrm{NH}_{4}$, Equação (6) Quadro 1, com o No, Equação (5) Quadro 1. Os resultados são apresentados no Gráfico 3. 


\section{Revista Nacional de}

Gráfico 3: Curvas do NTK obtida no Modelo Computacional de Fritz (1985).

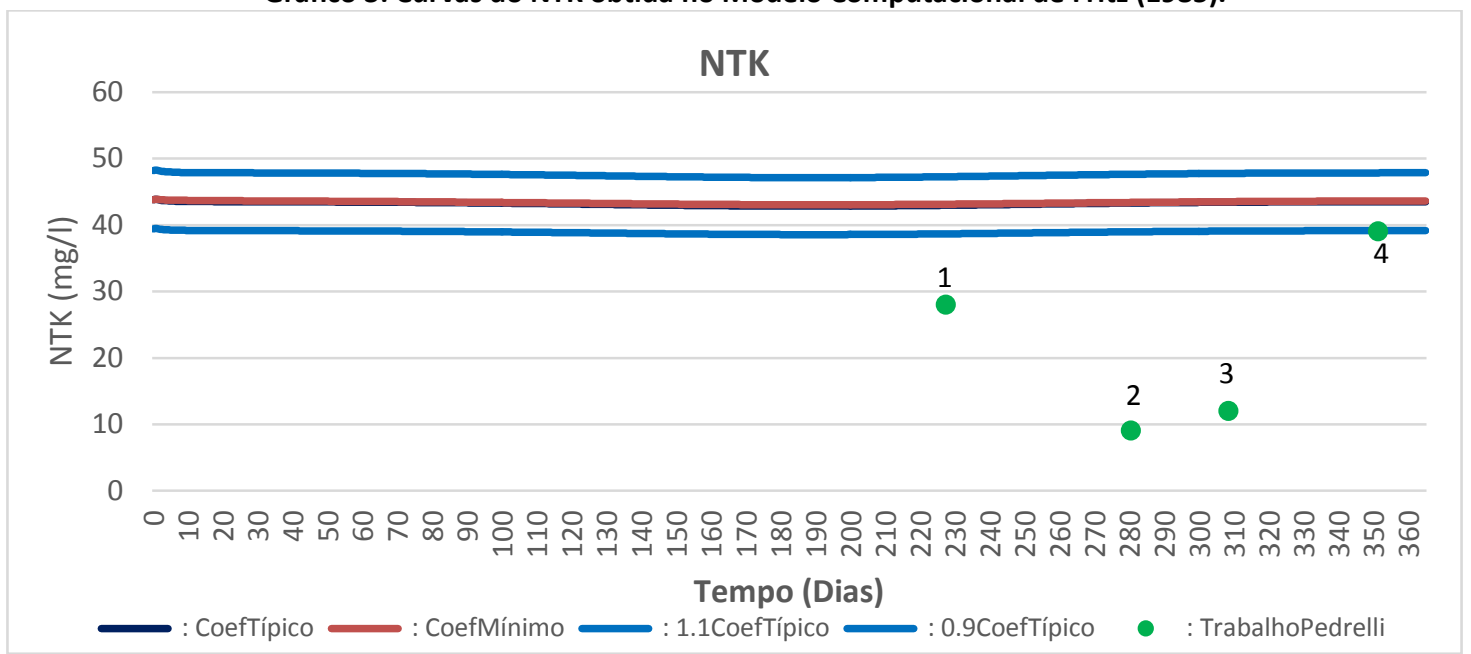

Fonte: AUTORES, 2018.

De acordo com o Gráfico 3, dos 4 (quatro) pontos que representam as amostras da lagoa facultativa, um está dentro do intervalo das curvas (Ponto 4) e os outros três estão abaixo. Então, 25\% das amostras apresentam-se dentro do que foi gerado pelo modelo e os outros $75 \%$ encontram-se com valores inferiores, ou seja, com a concentração real de $\mathrm{NH}_{4}$ no efluente menor do que a simulada. Assim como nos Gráficos 1 e 2, a curva de Coeficiente Mínimo e Coeficiente Típico são visualmente iguais.

\subsection{Concentração de Nitrogênio Amoniacal - $\mathrm{NH}_{4}$}

Calculado com base na Equação (6) do Quadro 1. As curvas geradas no modelo apresentam-se no Gráfico 4.

Gráfico 4: Curvas de NH4 obtida no Modelo Computacional de Fritz (1985).

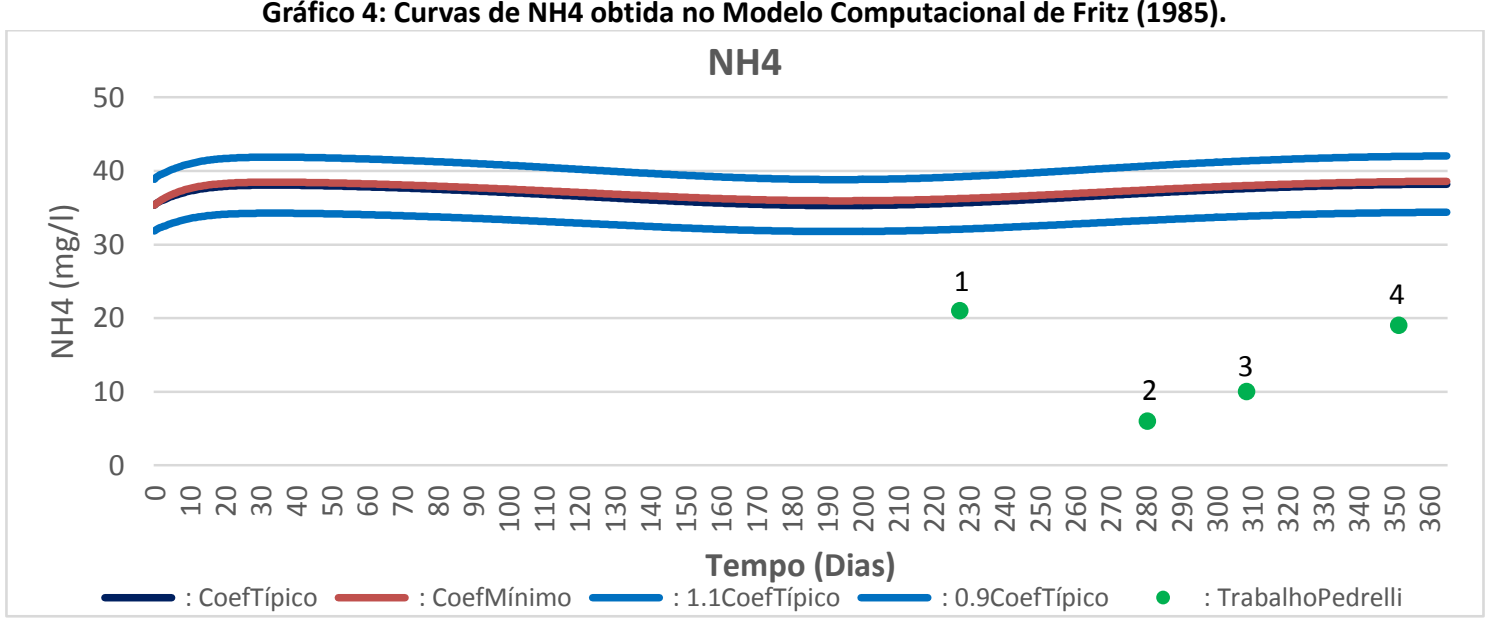

Fonte: AUTORES, 2018. 


\section{Revista Nacional de}

Conforme o Gráfico 4, pode-se afirmar que as curvas Coeficiente Típico e Coeficiente Mínimo não apresentam diferença visual, assim como os Gráficos 1, 2, 3. Dos quatro pontos obtidos do trabalho de Pedrelli (1997), nenhum entra em contato com o conjunto de curvas e todos encontram-se abaixo dele. Logo, a concentração real de $\mathrm{NH}_{4}$ do efluente é menor do que a obtida pelo modelo.

\section{CONCLUSÃO}

Com base no modelo matemático de Fritz (1985) foi possível desenvolver um modelo computacional na plataforma $\operatorname{VenSim}^{\circledast}$ capaz de simular o comportamento de lagoas facultativas.

Os testes efetuados para a calibração desse modelo mostraram que pode-se adotar tanto os valores típico quanto os valores mínimos estipulados pela literatura para os coeficientes bioquímicos, já que ambos apresentam resultados visualmente semelhantes pelo modelo.

Das análises efetuadas, aquela que apresentou valores mais satisfatórios foi a concentração do DQO solúvel, onde $68,4 \%$ dos pontos apresentavam-se fora do conjunto de valores. A grande quantidade de pontos fora do conjunto de curvas de concentração (S, St, NTK e $\mathrm{NH}_{4}$ ) pode ser explicada devido ao fato do modelo de Fritz (1985) considerar o modelo como uma mistura completa e com concentração de afluentes constante, ambas considerações inexatas quando se trata de lagoas facultativas.

No entanto, deve-se destacar que esse é um estudo ainda em desenvolvimento e que com base nos resultados obtidos no trabalho serão feitos novos ajustes no modelo buscando aproximá-lo do que é obtido na realidade.

\section{REFERÊNCIAS BIBLIOGRÁFICAS}

BRASIL. Ministério das Cidades. Secretaria Nacional de Saneamento Ambiental - SNSA. Sistema Nacional de Informações sobre Saneamento: Diagnóstico dos Serviços de Água e Esgotos - 2015. Brasília: SNSA/MCIDADES, 2017. 212 p. : il.

FERZIGER, Joel H.; PERIC, Milovan. Computational Methods for Fluid Dynamics. 3a ed. Berlin; Heidelberg; New York; Barcelona; Hong Kong; London; Milan; Paris; Tokyo: Springer, 2002. 423 p.

FRITZ, J. J. Mathematical Models for Waste Stabilisation Ponds. In: JORGENSEN, S. E.; GROMIEC, M.J. Mathematical Models in Biological Wastewater Treatment. ELSEVIER, New York. 1985. p. 169-241.

GONÇALVES, Julio Cesar de Souza Inácio. Desenvolvimento de modelo numérico para simulação da qualidade da água em rios utilizando o software Vensim PLE . 2009. 203 p. Dissertação (Mestrado em Engenharia Civil) Programa de Pós-Graduação em Engenharia Hidráulica de Saneamento, EESC-USP, São Carlos, 2009.

GRADY JR., C. P. Leslie et al. Biological Wastewater Treatment, Third Edition. 3. ed. Boca Raton: CRC Press, 2011. $962 \mathrm{p}$. 


\section{Revista Nacional de}

INSTITUTO BRASILEIRO DE GEOGRAFIA E ESTATÍSTICA (IBGE). Estimativas populacionais para os municípios e para as Unidades da Federação brasileiros em 01.07.2016. Atendendo ao dispositivo legal, Lei $n=8443$, de 16 de julho de 1992, no artigo 102, o IBGE fez publicar no Diário Oficial da União, em 30 de agosto de 2016, as estimativas de população para os municípios e para as Unidades da Federação brasileiros, com data de referência em 10 de julho de 2016. A Lei complementar no 143, de 17 de julho de 2013, alterou o art. 102 da Lei no 8443, revogando os parágrafos 1 을 e 2 , que estabelecia o prazo de 20 dias após a publicação das estimativas no Diário Oficial da União para apresentação de reclamações ao IBGE. Diário Oficial da União, Poder Executivo, Brasília, DF, 30 Ag. 2016.

KELLNER, Erich; PIRES, Eduardo Cleto. Lagoas de estabilização: projeto e operação. Rio de Janeiro: ABES, 1998. $244 p$.

MARA, Duncan; HORAN, Nigel. Handbook of water and wastewater microbiology. 1 ed. London: Academic Press, $2003,832 \mathrm{p}$

METCALF \& EDDY, INC. Wastewater engineering treatment and reuse. 4a ed. New York: McGrawHill, 2003. 1819 p.

PEDRELLI, Tânia Denise. Avaliação do Sistema de Lagoas de Estabilização para o Tratamento das Águas Residuárias de Balneário Camboriú/SC. 1997. 154 p. Dissertação (Mestrado em Engenharia Ambiental) - Programa de PósGraduação em Engenharia Ambiental, UFSC, Florianópolis, 1997.

TRUPPEL, Anderson. Redução de Odores de uma Lagoa de Estabilização de Esgoto Sanitário e Avaliação da Qualidade de seu Efluente. 188 p. 2002. Dissertação (Mestrado em Engenharia Ambiental) - Programa de PósGraduação em Engenharia Ambiental, UFSC, Florianópolis, 1997.

VON SPERLING, Marcos. Biological Wastewater Treatment Series: Waste Stabilization Ponds. Volume 3. London: IWA Publishing, 2007. $162 \mathrm{p}$. 